\title{
NOTES \\ SUR LA CONSERVATION DES SOLS AUX ETATS-UNIS
}

\section{I. - Histoire de l'utilisation du sol}

L'histoire de l'utilisation du' sol par l'homme est toute récente aux Etats-Unis et peut être assez aisément retracée, car le tapis végétal primitif a subi ses modifications les plus importantes au moment de l'afflux massif des colons aux $\mathrm{XVII}^{\ominus}$, XVIII' ${ }^{\theta}$ et $\mathrm{XIX}^{\boldsymbol{\theta}}$ siècles.

Les descriptions des premiers explorateurs montrent des Indiens suivant dans leurs migrations les troupeaux de bisons dont ils tiraient leur subsistance et qui, par conséquent, n'avaient guère modifié le paysage naturel. Leurs cultures ne couvraient pas de grandes étendues, et, s'il est difficile de dire quelle a été l'importance des incendies qu'ils avaient allumés pour accroitre les terrains de parcours destinés à l'élevage du bétail acquis des Espagnols, il est certain que, jusqu'aux $\mathrm{XVII}^{\mathrm{e}}$ et $\mathrm{XVIII^{ \textrm {e } }}$ siècles, le territoire actuel des EtatsUnis était surtout couvert de prairies et de forêts.

\section{XVII ${ }^{\theta}$ ET XVIII' SIÈCLES}

Les premiers colons arrivent en Virginie en I607. Les pèlerins du Mayflower débarquent en I620. A cette époque, la forêt vierge s'étend sur toute la côte atlantique, du Maine à la Floride. Les forêts de Pensylvanie en particulier, qui donnent son nom à 1'Etat fondé par William PENN, regorgent de gibiers de toutes sortes.

Le courant d'émigration vient d'Europe, surtout de Grande-Bretagne. La France a constitué à cette époque ın empire colonial qui s'étend dı Saint-Laurent à la Nouvelle-Orléans, et les hostilités entre les établissements français et les Treize Colonies anglaises de la Côte Atlantique commencent dès I6r3. Celles-ci entrèrent en rebellion contre l'Angleterre en I775, et leur indépendance est reconnue par les traités de $I 783$.

A ce moment, il n'y a guère que 4000000 d'habitants aux EtatsUnis, dont 250000 seulement dans ce qu'on appelle alors " l'O11est ») le Vermont, le Kentucky, le Tennessee, l'Ohio. 
En dehors de la zone atlantique Nord des Etats-Unis, l'utilisation des sols est donc très récente. Quelques repères historiques permettent de le préciser: la cession de la Louisiane par la France date de I803, la cession de la Floride de I8I9, celle de l'Oregon de I846, celle des territoires mexicains de I848, celle du Texas de I850, celle de l'Alaska de I867.

Ainsi, la colonisation s'est produite avant la période de modernisation et de progrès technique qui a transformé l'agriculture européenne au $\mathrm{XIX}^{\theta}$ siècle. C'est pourquoi les techniques agricoles des premiers colons se révélèrent maladroites et inadaptées, soit que les connaissances de la plupart d'entre eux aient été insuffisantes, soit qu'ils aient cherché à appliquer les routines de leurs pays d'origine, sans se rendre compte des différences imposées par le sol ou le climat. La soif d'enrichissement rapide, qui est une des caractéristiques de l'esprit pionnier, les conduisait à traiter la terre sans ménagement, cherchant à en tirer rapidement le maximum.

Les effets de la colonisation ne tardèrent pas à se faire sentir, bien que les défrichements des premiers arrivants n'aient été massifs qu'en certains points, et que la chasse ait souvent été pour eux un moyen d'existence plus important que la culture. Dès I68I, William Penn, fondateur de la Pennsylvanie, prescrivait de laisser un acre de forêt pour cinq acres défrichés. Cette tentative ultime pour conserver au paysage de cet Etat son renom forestier aboutissait à un échec. A la fin $\mathrm{XVII}^{\circ}$ siècle, les modifications du climat de la côte atlantique sous l'effet du déboisement massif étaient évidentes: les inondations se faisaient plus fréquentes, les eaux des rivières avaient perdu leur limpidité et charriaient les débris de l'érosion. Il est vrai qu'au même moment le commerce du tabac, celui de la térébenthine, extraite des vastes forêts de pins du Sud, de la potasse, tirée des cendres de bois, et l'industrie des constructions navales permise par l'exploitation des arbres centenaires, florissaient en Nouvelle-Angleterre, dans les Carolines et en Virginie: exemple d'utilisation massive des ressources de la Nature sans souci du lendemain.

Vers I740, la colonisation gagne le Sud-Est des Appalaches, zone de piémont, couverte de verdoyantes prairies naturelles, qui est livrée à la monoculture épuisante du coton, pratiquée sans assolement. L'année I793 est marquée par l'invention du " cotton gin », machine à égréner le coton, qui donne une nouvelle impulsion à cette culture. Ce début de mécanisation accélère l'élaboration de la structure sociale du Sud: on achète les terres des fermiers les moins fortunés, les “ pauvres blancs », qui s'en vont cultiver les pentes des Appalaches, tandis que se constituent de grandes plantations à main-d'œuvre noire. Les premiers esclaves ont été débarqués en I6Ig et le fameux trafic triangulaire a sévi aux $\mathrm{XVII}^{\mathrm{e}}$ et $\mathrm{XVIII}^{\mathrm{e}}$ siècles. L'emploi de cette main-d'œuvre peu instruite ne devait pas améliorer les méthodes d'utilisation des sols. 


\section{SIÈCLE}

Au début du $\mathrm{XIX}^{\mathrm{e}}$ siècle, les effets de l'érosion dans le Sud étaient évidents. Les tempêtes de poussière n'étaient pas rares. Les lettres des contemporains, les écrits de Washington et de JefFerson entre autres, renferment des indications caractéristiques: le lessivagac des sols, le ravinement y sont décrits en détail. Le Caroline du Sud, en proie aux inondations provoquées par le défrichement des pentes, devint le pays de la Malaria.

Après une longue période de stagnation technique, marquée par l'emploi de méthodes médiocres et primitives, - puisque, jusqu'en I8I9, il n'y avait pas d'autre assolement dans le Nord-Est que la succession grain-herbe-jachère -, l'agriculture américaine devint plus industrielle, mais aussi plus meurtrière pour le sol, la force brutale et puissante de la machine se substituant au travail de l'homme. L'invention de la moissonneuse Mac Cormick date de I83I. La charrue d'acier a été mise au point en I837. A partir de I830 s'amorce un mouvement important de colonisation à l'Ouest du Missouri. Le Gold rush Californien date de 1948. Le Nord de la Prairie Centrale a été colonisé aux environs de I860, après l'achèvement du Canal de l'Erié et l'établissement de la navigation sur les Grands Lacs. Une immigration massive au Sud de la Prairie et vers la côte Ouest s'est produite aussitôt après la Guerre civile (I866), grâce an développement rapide des chemins de fer.

Les Grandes Plaines du Centre des Etats-Unis étaient alors couvertes d'une admirable prairie naturelle, domaine des troupeaux de bisons, vivant sur une association végétale climacique ou paraclimacique constituée de nombreuses graminées vivaces. La prairie n'avait été que peu modifiée par l'introduction du cheval amené par les conquistadors espagnols, et que les Indiens avaient appris à utiliser pour la chasse des bisons. La destruction massive des millions de bisons qui la peuplaient, et le refoulement corrélatif des Indiens, s'est effectué de I870 à I88o. Puis vint la mise en culture de blé d'une partie de ce territoire, mise en culture spéculative décrite sous le nom de "Wheat rush ), bientôt suivie d'une extension menaçante des phénomènes d'érosion. En même temps les troupeaux de Longhorn, bétail peu amélioré dont l'origine remonte aux bovins introduits par les Espagnols, remplaçaient les troupeaux de bisons voués à la disparition. Bientôt, c'était le boom de l'élevage, avec l'invention, en I885, du fil barbelé de clôture, qui rendait possible le surpâturage.

La colonisation de l'Ouest entraîna une transformation du milieu naturel: de nombreuses graminées disparurent de la liste des espèces végétales de la Grande Prairie. On assista d'abord à l'appauvrissement en espèces de la flore. Grâce au témoignage des botanistes et agronomes de l'époque, on sait par exemple qu'au Kansas, les pâturages qui, à l'origine, étaient constitués d'un mélange de graminées 
vivaces dont Buchloe dactyloïdes et divers Andropogons, ne continrent bientôt plus que des Buchloe qui, à leur tour, firent place à des graminées annuelles.

Dans l'Arizona, la prairie comprenait, outre les Boutelouea et Sporobolus, des Hilaria qui nourrissaient les troupeaux dès le printemps, et des Salvia qui constituaient le fourrage d'arrière-saison. Il y eut d'abord appauvrissement dı mélange. puis apparition de graminées annuelles, dont certaines d'introduction récente. Dans ces conditions, il suffit de deux hivers exceptionnellement rigoureux, en 1885 et I 887 , pour détruire complètement les ressources fourragères et mettre un point final au boom de l'élevage.

La brutalité destructrice de la colonisation de peuplement s'explique en partie par un mode de répartition des terres qui ne tenait aucun compte de la vocation ou de la qualité du sol. Le quadrillage cadastral rectangulaire, institué en 1785 , qui couvre une bonne partie des Etats-Unis et fait songer au quadrilatère romain, est parfaitement rigide et méconnait même le relief. Il servit de base au système de vente par unités de I mille carré établi à cette époque. A partir de 1832 , l'étendue des lots fut de 40 acres, puis de 80 acres. En fait de nombreux colons s'étaient installés sans titre sur les terres vacantes. En I84I, la loi permit l'acquisition des terres du domaine public au prix de 1,25 \$ l'acre. Cette condition devait être jugée trop dure, et, en I862. le Homestead Act permit l'appropriation gratuite des terres vacantes sous réserve de leur mise en culture et d'une durée minima de résidence. Pratiquement, l'application du système donna lieu à de nombreuses fraudes. Certains spéculateurs réussirent à accaparer des lots étendus et à constituer de grands domaines. Alors que le Homestead Act n'attribuait la terre que par lots de I60 acres, les colons eurent bientôt la faculté d'obtenir deux lots chacun. De I873 à I89I, il fut possible de bénéficier de 480 acres par personne dans les Etats neufs, et les moyens de tourner la loi étaient nombreux.

De I862 à I87I fut mis en application le système des « Railroad grants », concessions de larges bandes de terrain faites aux Compagnies de chemin de fer pour qu'elles y établissent une voie ferrée en se payant par la vente des lots ainsi valorisés. Ce système a donné lieu, bien entendu, à la naissance d'activités agricoles spéculatives, et certains de ces " grants ) comme ceux qu'on appelle les “ matvaises terres ») du Wyoming, sont encore aujourd'hui des exemples remarquables de sols érodés.

En I878, le Timber and Stone Act facilitait l'attribution aux colons, à un prix très modique, de lots de 160 acres pour l'exploitation de forêts ou de carrières. On voit qu'il n'était pas encore question de politique forestière. 


\section{DÉBUT DU XX⿳⺈ SIËCLE}

Le système d'attribution des terres visait à la création d'une nation de petits cultivateurs groupés en communautés rurales. Mais ce but ne fut pas atteint. Il y eut en fait concentration de la propriété, et bientôt de nombreux possesseurs de terres ne cultivèrent plus eux-mêmes. Dès I9ıo, il y avait $42 \%$ de fermiers parmi les cultivateurs et, comme de nombreux lots ne pouvaient constituer des unités rentables d'exploitation, on assista à la création d'un prolétariat agricole.

Alors qu'à l'Est du Mississipi la propriété privée domine, à l'Ouest, de nombreux terrains sont restés dans le domaine public. En effet, à l'Ouest de la Prairie Centrale, le pays devient désertique et improductif, et de nombreux lots n'ont pas trouvé d'acquéreenrs. L'activité normale est l'élevage, mais les lois sur le settlement ne permettaient guère de rassembler une étendue suffisante pour l'établissement d'une ferme d'élevage. On prit donc l'habitude de faire paitre les animaux sur les terres du domaine public, comme chez nous sur des terrains communaux, avec cette différence que chaque éleveur se réservait un secteur autour de son ranch. Le domaine public a donc conservé une étendue considérable. Il représente $44 \%$ de la surface du New-Mexico, $73 \%$ de celle de l'Arizona, $85 \%$ de celle du Nevada. Mais pour être inapproprié, il n'en a été utilisé que plus abusivement: il a été en fait livré, au moment du boom de l'élevage, au surpâturage généralisé. Ainsi s'est constitué un paysage désertique et érodé, le " range ", véritable brousse, caractéristique des plateaux arides de l'Ouest. Les exploitations typiques sont les " ranches ", fermes d'élevage, qui font quelques cultures de fourrages d'hiver et utilisent le " range " comme terrain de parcours. On estime que ce dernier a perdu, depuis l'origine, 50 à $70 \%$ de sa capacité fourragère.

Suivant les principales étapes que l'on vient d'indiquer, la colonisation a progressé d'Est en Ouest, l'utilisation des terres de l'Ouest étant toute récente. Chose remarquable. les fermiers de l'Ouest ne furent bien souvent que ceux-là même qui venaient d'abandonner les exploitations ravagées par l'érosion à l'Est. La possibilité de trouver de nouvelles terres à l'Onest au fur et à mesure de l'expansion politique de la Fédération, explique l'abandon rapide des terres, souvent après quelques années de culture. Elle rend compte d'une caractéristique essentielle des paysages de l'Est: la présence de terres abandonnées par la culture. couvertes d'une forêt secondaire récente qui commence à guérir les blessures anciennes de l'érosion.

Mais le mouvement vers l'Ouest devait, de toute évidence géographique, avoir une fin. De I830 à 1934, on en vint à vendre les terres réservées à l'origine aux Indiens par traités, et jugées inutiles 
à leur subsistance. Les réserves indiennes de l'Oklahoma furent les dernières ouvertes à la colonisation, de I889 à I906. L'Oklahoma rush, course collective des colons, qui avaient pris le départ en ligne pour la conquête des dernières terres vacantes, fut l'acte final, et non le moins pittoresque, de la marche vers l'Ouest. C'est alors, qu'au début du $\mathrm{xx}^{\mathrm{\theta}}$ siècle, le mouvement de migration vers les villes, grossi du reflux des fermiers parvint à son maximum. En même temps, on se mit à couper et défricher ce qui restait de forêt primitive dans les territoires de l'Est. Ainsi l'exploitation massive des forêts du Wisconsin eut lieu de I880 à I9IO, puis. les sols forestiers défrichés furent livrés à la culture On chercha en dernier ressort à rendre de nouvelles terres productrices par l'irrigation.

La première guerre mondiale, par le regain d'activité qu'elle donna à l'agriculture des Etats-Unis, accrut encore le rythme de l'usure des sols. Les tempêtes de poussière, qui marquent le début de la formation des dunes continentales sóus l'action de l'érosion, coïncidèrent avec la dépression économique des années 1930 et le mouvement de' retour au sol qu'elle avait provoqué. Ces nuages sinistres de limon obscurcissant le ciel impressionnèrent l'opinion publique. Il apparut alors que le maintien du sol en bon état était un problème de gouvernement.

\section{II. - La politique de conservation du sol}

L'opinion prit brusquement conscience du fait que le sol, comme le pétrole ou les ressources minières, est une richesse qu'il importe de ne pas gaspiller si l'on veut assurer l'avenir du pays. C'est l'origine de la politique de Conservation, patronnée par Franklin D. Roosevelt.

\section{Le « Resettlement 》 et Le “ Zoning »}

Les remèdes appliqués ont été nombreux et efficaces, à la manière américaine. L'effort du Gouvernement s'est traduit tout d'abord par la création du Civilian Conservation Corps, destiné à l'exécution de grands travaux de lutte contre le chômage. Les camps de travailleurs C.C.C., comme on les a appelés, sont restés célèbres par leurs réalisations : routes, digues, ouvrages d'art, reboisements. Nous verrons comment ils ont été à l'origine de la mise en valeur de la vallée du Muskingum dans l'Ohio. Ils ont permis aussi de très belles réalisations dans les Parcs nationaux et les forêts fédérales.

En matière de conservation des sols proprement dite, la création la plus originale, la plus hardie, mais aussi la plus discutée, a été, en 1935, celle de l'Administration du "Resettlement ", mot que l'on pourrait traduire par réinstallation. L'idée directrice était de déplacer les fermiers qui se trouvaient sur des terres trop pauvres pour leur procurer un niveau de vie considéré comme minimum. 
Cette idée est basée par conséquent sur la théorie des terres « submarginales »: insuffisantes pour procurer une rémunération comparable à celle fournie par les autres secteurs de l'économie nationale et devant, par conséquent, être abandonnées par la culture pour être transformées en pâtures et en forêts, entreprises moins exigeantes en main-d'œuvre.

On estimait à l'époque que 500000 familles rurales devaient être réinstallées sur des terres neuves prélevées sur le domaine public. Le système, qui supposait l'achat par l'Etat des terres improductives pour l'agriculture, comportait un programme d'acquisition imposant, portant sur 650000 fermes pour une surface de plus de 40000000 d'hectares. Il reçut un commencement d'exécution: 360000 hectares furent achetés, pour une dépense d'environ I7 000000 \$. Là guerre interrompit cette expérience qui donna lieu aux plus vives critiques et à une opposition massive au sein du Congrès, tant en raison des sommes dépensées qu'en raison des protestations des personnes déplacées d'office.

Très voisine de la politique du ("Resettlement ), celle du ("Zoning ) a été mise en œuvre à la même époque dans certains secteurs de l'Est des Etats-Unis et particulièrement dans le Wisconsin, où de nombreuses fermes avaient été abandonnées à partir de 1924, pendant la période de dépression économique. Alors quel la politique de " $R e$ settlement » a été surtout appliquée dans le Middle-West, pour réparer les erreurs commises lors de l'établissement des exploitations, le Zoning s'applique à un peuplement moins récent. Il consiste à déterminer les zones où l'établissement de fermes isolées est jugé peu souhaitable. Il s'agit de supprimer les dépenses à la charge de la collectivité qui apparaissent trop lourdes lorsqu'il faut desservir une poussière de fermes, par exemple la voirie, l'adduction d'eau et d'ẻlectricité, le transport des enfants à l'école et autres services publics. Comme les fermes trop isolées sont souvent peu rentables, on espère aussi éviter l'extension des terres abandonnées sur lesquelles l'Etat ne peut plus récupérer d'impôts. Selon la loi, ces terres font retour à l'Etat qui ne peut plus guère les utiliser que par le boisement.

Bien entendu, le zoning doit tenir compte du danger d'érosion de la menace qui peut résulter pour le régime des eaux de la mise en culture inconsidérée de pentes trop abruptes ou affouillables. Dans les zones ainsi déterminées et qui comprenent la plupart des terres pour lesquelles l'impôt foncier n'est plus payé depuis un certain nombre d'années, et redevenues par conséquent propriété de l'Etat, il est interdit de s'établir à nouveau en cas de cessation d'exploitation. Cette politique est donc beaucoup plus souple que celle du resettlement. Il ne s'agit pas de modifier immédiatement l'utilisation du sol, mais d'organiser son utilisation future; l'influence des mesures prises sur la valeur marchande des terrains n'en est pas moins 
évidente. En pratique, un registre des établissements non conformes au zoning est établi; pour cetıx-là, toute reprise d'exploitation est interdite. En outre, l'Etat ou le Comté ont la faculté de proposer aux intéressés des échanges de terrain pour faciliter leur réinstallation dans des conditions conformes au zoning. Ce système qui n'a pas donné lieu à des difficultés majeures est encore en application.

\section{Le “Soll Conservation Service »}

Dans cette période des années I930, la création gouvernementale la plus importante a été sans aucun doute celle du Soil Conservation Scrvice qui a donné toute son ampleur au mouvement de Conservation des sols. Il a été précédé par le Bureau de l'Erosion, créé en I933 et dépendant du Ministère de l'Intérieur, qui a établi dans presque tous les Etats des zones de démonstration. Puis ce bureau est devenu en 1935, le service de Conservation des sols, rattaché.au Ministère de l'Agriculture. Son action est marquée dès le début par deux caractéristiques essentielles: importance donnée à la propagande et à l'enscignement, organisation ramifiée sur le terrain en " districts " de structure démocratique.

Il s'agit de fournir aux exploitants agricoles les renseignements techniques, et aussi les moyens financiers qui leur permettront d'utiliser au mieux les terrains dont ils disposent. Il n'est pas inutile de préciser que les exploitants agricoles qui se conforment aux indications données par le Soil Conservation Service reçoivent l'aide maxima des divers organismes qui distribuent le Crédit Agricole: Production and Marketing Administration, Farm Credit Administration, Farm Home Administration. Commodity Credit Corporation.

Le Soil Conservation Service établit pour tout exploitant qui en fait la demande une carte de son domaine indiquant les possibilités des divers types de sols qu'on $\mathrm{y}$ rencontre. Cette carte est réalisée sur le canevas d'une photographie aérienne à grande échelle d'après des relevés pédologiques permettant le repérage des divers profils de sol. En fonction du degré d'érosion, des caractéristiques du climat, de la sécheresse ou de l'humidité, des cultures habituelles dans la région considérée, on classe les sols dans les catégories de " vocation " établies à l'avance (land capability classes). Ces catégories sont au nombre de 8:4 correspondent aux terres cultivables avec des précautions d'importance croissante, de I à 4. Ainsi, les meilleures terres rentrent dans la catégorie $\mathrm{n}^{0} \mathrm{I}$, les terres dont la mise en culture est à la rigueur possible, quoique présentant de graves difficultés résultant de l'érosion, de la sécheresse, ou du manque de drainage, dans la $4^{8}$ catégorie. Les catégories 5,6 et 7 correspondent aux sols dont il vaut mieux abandonner la culture pour les transformer en pâtures ou en forêts. Autrement dit, ce sont les 
sols qui doivent bénéficier d'une couverture végétale permanente pour éviter la dégradation par l'érosion, ou qui sont trop humides ou trop secs pour la culture. Dans les catégories 6 et 7 , on range les sols où pâture et forêt ne pourront être maintenues qu'au prix de précautions de plus en plus importantes. La catégorie 8 comprend les sols dégradés ou les marais qu'il serait vain d'essayer de cultiver, où prairie ou forêt ne petıvent donner de bons résultats. On estime généralement que leur seule utilité consiste à fournir un milieu favorable au gibier et aux animaux sauvages.

Il s'agit donc d'une systématisation de l'utilisation du sol, utile dans un pays neuf où les agriculteurs sont exposés à commettre un certain nombre d'imprudences et d'erreurs. Ces cartes des possibilités des sols ont été rapidement très appréciées des intéressés.

Par ailleurs, dès son origine, le Soil Conservation Service a pris à cœur de rendre populaire l'idée de conservation du sol, et de diffu-• ser largement les techniques qu'il préconise. De nombreuses conférences, des films, des opuscules ont informé le grand public de la nécessité de conserver le sol, considéré comme un patrimoine national et comme le support du développement futur du pays. Cette propagande a été renforcée par l'action de plusieurs associations privées, des clubs, des ministres des cultes, et de la plupart des universités.

Le Service de Conservation du sol groupe aujourd'hui $80 \%$ au moins des cultivateurs des Etats-Unis dans ce qu'on appelle les " districts de Conservation ». Chacun est constitué par les cultivateurs d'une petite région naturelle. Des assemblées de fermiers élus se réunissent périodiquement pour établir le plan des améliorations agricoles nécessaires dans la région, et discuter les mesures préconisées, dont aucune n'est d'ailleurs imposée obligatoirement aux participants - quelque chose en somme comme les Centres d'études techniques agricoles qui prennent naissance en ce moment dans notre pays. En I950, il y avait environ 2200 districts de Conservation du sol aux U.S.A. couvrant $75 \%$ des terres cultivables, soit une superficie évaluée à 440000000 d'hectares. Le district moyen couvre donc 200000 hectares et groupe 2000 cultivateurs.

Le Soil Conservation Service met un agent à la disposition de chaque district pour préparer et mettre au point les cartes des possibilités des sols et les plans d'amélioration. Les agents des districts sont placés sous l'autorité d'un bureau central dans chaque Etat. A l'échelon supérieur, il y a sept bureaux régionaux remarquablement équipés pour le tirage des plans ou des photographies aériennes, et qui possèdent des spécialistes avertis des questions agricoles. Au total, le Soil Conservation Service groupe près de I4 0oo agents.

L'œuvre réalisée par le Soil Conservation Service a une remarquable ampleur. Dans leur ensemble, les phénomènes d'érosion ont été arrêtés, de vastes étendues remises en valeur. Par les techniques 


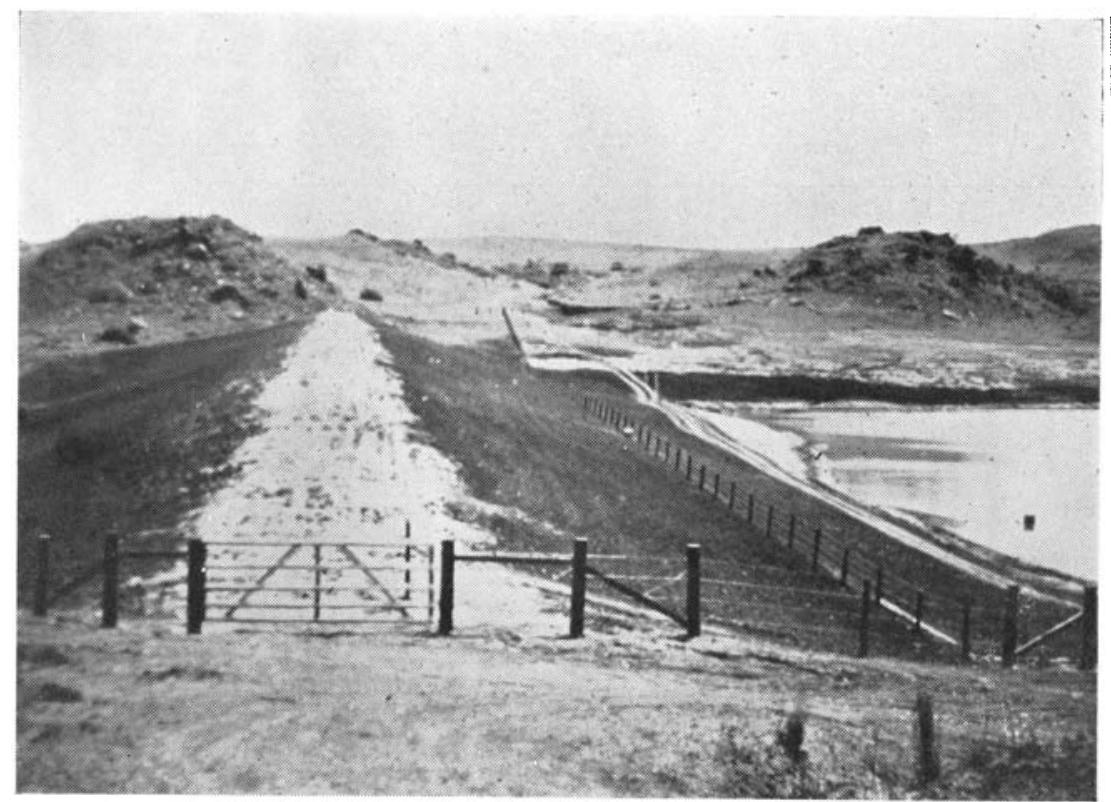

I.utte contre les inondations. Barrage de retenue en terre (Oklahoma).

(Cliché Soil Conseration Service.)

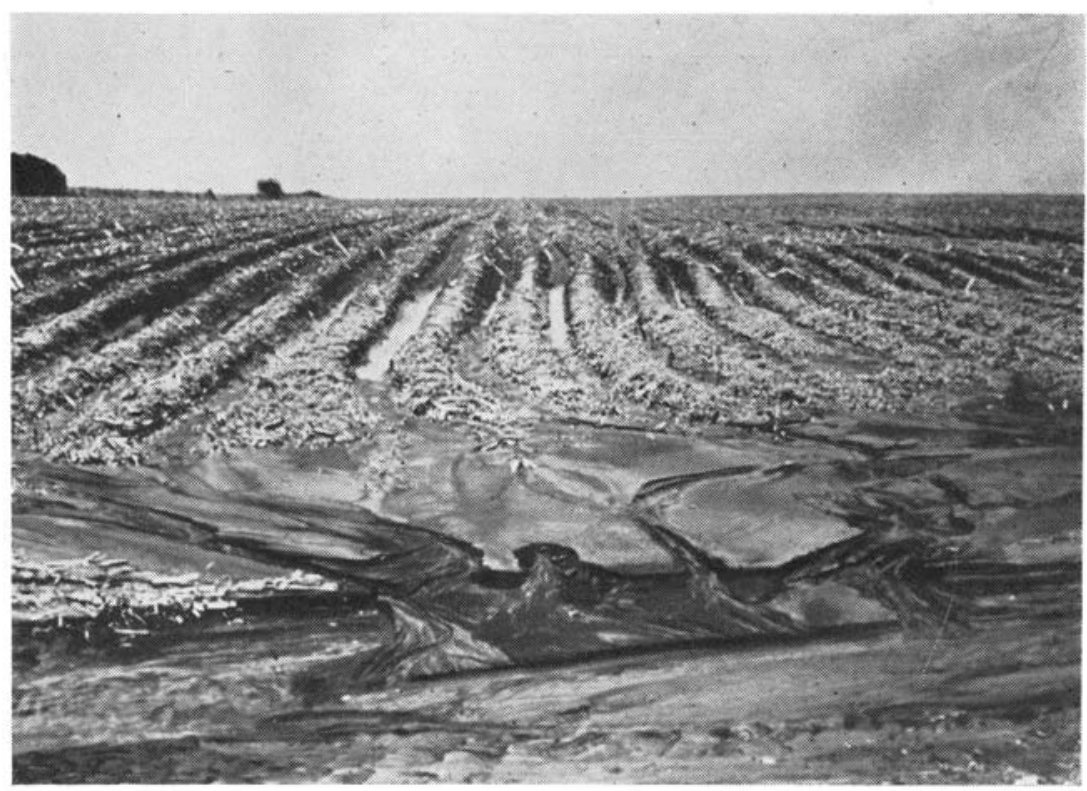

Erosion dans un champ cultivé suivant la ligne de la plus grande pente (Nebraska).

(Cliché Soil Conseriation Service.) 


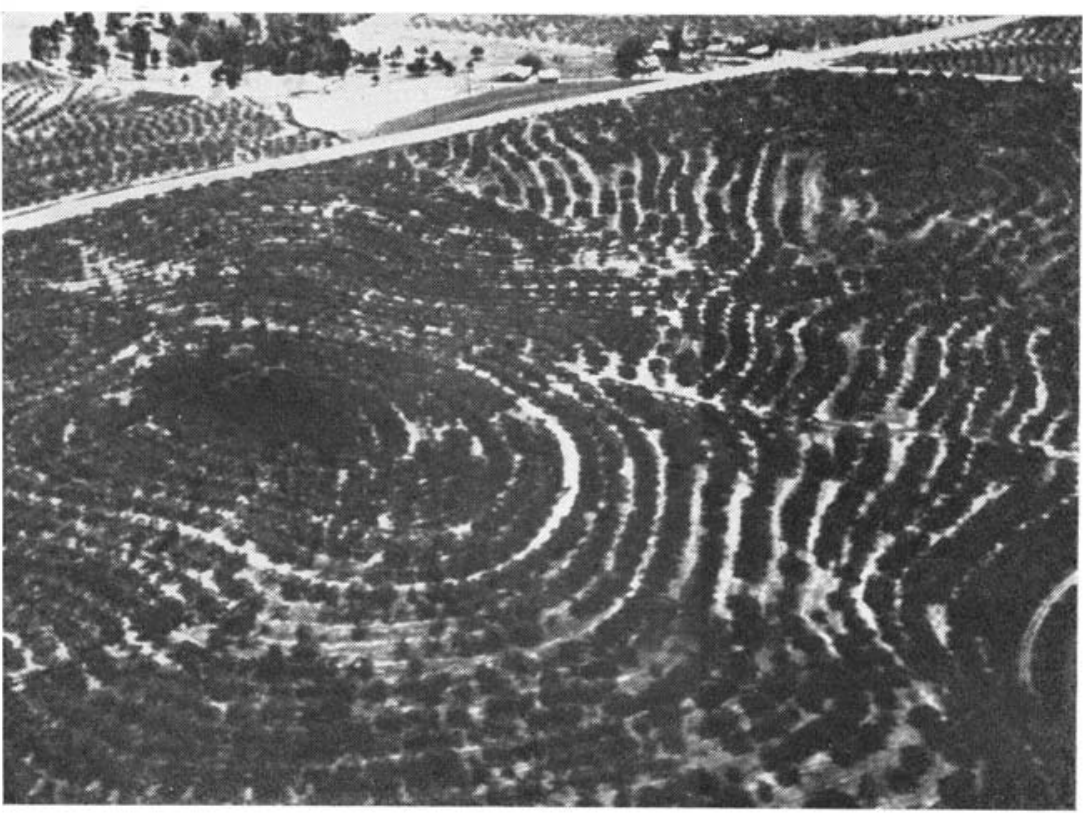

Verger de péchers établi en suivant les courbes de niveau. (Caroline du Sud).

(Cliché Soil Conseration Service.)

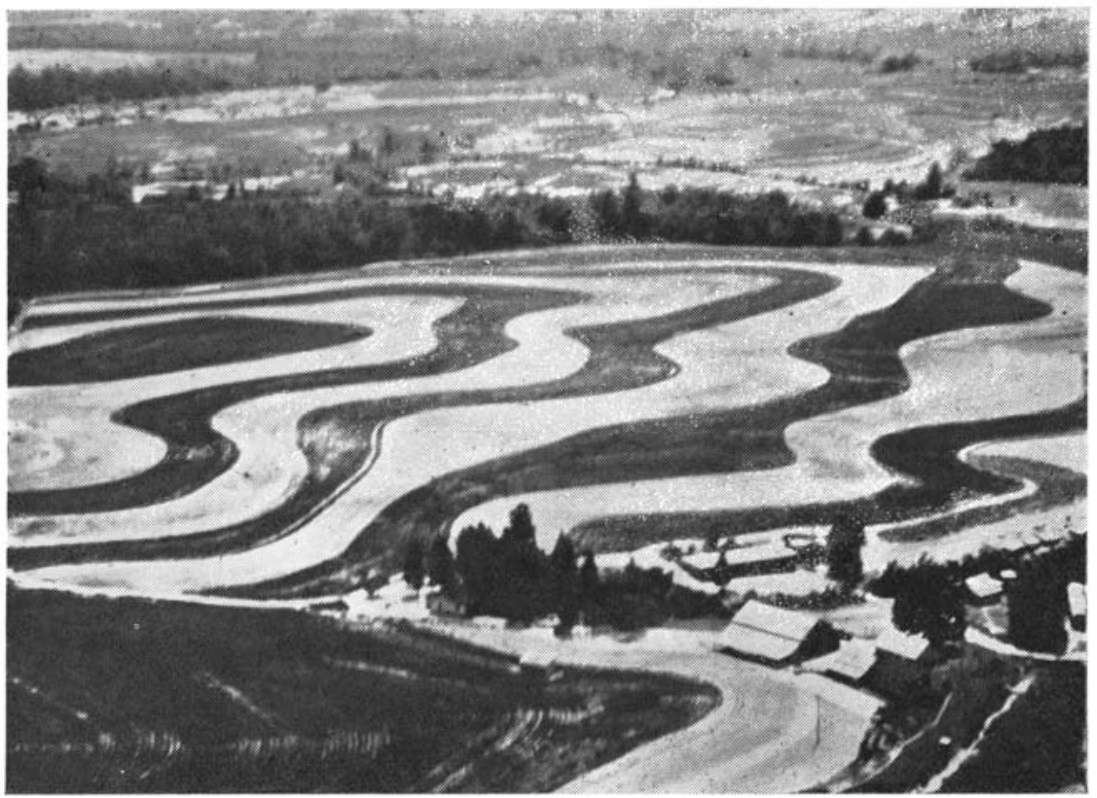

Culture suivant les courbes de niveau (Caroline du Sud).

(Cliché Soil Conservation Service.) 
qu'il a mises au point et préconisées, on peut dire qu'il a marqué le paysage de son empreinte. Il a généralisé les labours suivant les courbes de niveau sur les terrains en pente, précaution essentielle contre l'érosion dans un pays de pluies brutales. En pays sec, ils permettent de retenir l'eau bien mieux que les labours suivant les lignes de pente. Sur les pentes assez fortes, les labours en courbes de niveau alternent avec des bandes gazonnées, ou des bandes de cultures différentes alternent entre elles, permettant de ralentir le ruissellement et de retenir le sol. Cette pratique, qui donne un aspect original à la campagne américaine, est maintenant très généralement adoptée. Sur les terrains largement vallonnés, lorsque la pente ne dépasse pas ıo \%, on établit des banquettes, dont la base mesure une dizaine de mètres, la hauteur 40 à $50 \mathrm{~cm}$. La pente de la partie amont de la banquette, qui collecte l'eau, est de 4 à $8 \%$. L'eau s'écoule vers un chenal d'écoulement gazonné pour éviter toute érosion. Sur les pentes supérieures à Io \%, les banquettes sont considérées comme d'établissement trop onéreux, et susceptibles de céder sous de fortes averses. Elles sont d'ailleurs d'une façon générale moins utilisées qu'il y a une dizaine d'années.

Le Soil Conservation Service a eu aussi une influence considérable dans la vulgarisation de l'emploi des engrais, l'adoption de rotations, des cultures dérobées permettant de maintenir un certain couvert, la généralisation de l'irrigation, du drainage, et de nombreuses autres améliorations. Dans la Prairie Centrale, ò̀ l'érosion causée par le dry farming avait abouti aux " dust bowls », il a mis au point un système de culture basé sur des sous-solages, avec maintien sur le sol d'une couverture protectrice de chaumes ou autres débris abandonnés au moment des récoltes. C'est ce qu'on appelle le "stubble-mulch farming ", dont le but est d'accroitre la perméabilité de la surface du sol et de diminuer l'évaporation. En même temps a été établi un réseau de rideaux d'arbres. les " shelterbelts "), destinés à briser l'action des vents.

\section{Le « Bureau of Land Management »}

De la période qui a précédé la seconde guerre mondiale datent les premières mesures de protection des terres de l'Ouest restées dans le domaine public et bien souvent livrées au pâturage abusif. Le Taylor Grazing Act de 1934 a permis au Ministère de l'Intérieur de créer des Districts de Pâturage (Grazing districts), gérés par des bureaux où siègent côte à côte des représentants de l'Administration et des éleveurs. Grâce à cette institution, on a pu limiter le surpâturage et faire adopter certaines techniques de lutte contre l'érosion.

Bien que la propagande du Bureau of Land Management, chargé de la gestion des terres du domaine public au Ministère de l'Intérieur, ait un rayonnement moindre que celle du Soil Conservation 
Service, et qu'elle se heurte à une certaine opposition des éleveurs de l'Ouest, quelques travaux d'amélioration ont pu être effectués. malheureusement limités par le manque de crédits, sur les $68 \mathrm{mil}$ lions d'hectares de terrains de parcours dans les onze Etats de l'Ouest dont ce service à la charge.

Des techniques intéressantes ont été mises au point pour la lutte contre l'érosion dans les pâturages des régions sèches, en particulier des digues destinées à répartir les eaux de ruissellement en les empêchant de suivre la ligne de pente, et à les détourner des ravines, des sillons établis suivant les lignes de niveau pour freiner l'érosion, des obstacles constitués de cordons de pierres amoncelées ou de branches, espacés de Ioo à 300 mètres sur les pentes les plus douces, et qui, suivant les lignes de niveau, ont pour objet de retarder les eaux de ruissellement et de retenir les particules fines du sol qu'elles entraînent. Ces dispositifs évoquent, avec moins de relief, les banquettes de la D.R.S. Algérienne.

Mais la clef de la gestion des pâtures pauvres et dégradées des pays arides de l'Ouest des Etats-Unis est l'amélioration des méthodes de pâturage: réduction de la charge en bétail, rotations saisonnières, et répartition correcte des animaux qu'il importe de ne pas laisser trop longtemps à la même place. Dans ce domaine, l'action des Grazing districts a été fort importante.

\section{III. - Quelques exemples de réalisations}

Voilà comment se pose, esquissé à grands traits, le problème de Conservation des Sols aux Etats-Unis et quels remèdes ont été apportés à une situation qui, en I930, apparaissait comme très inquiétante. Ces remèdes, de même que les diverses méthodes qui ont été élaborées, ne nous intéressent pas toujours directement en tant que forestiers. Mais une partie de l'œuvre accomplie est susceptible de nous apporter des enseignements, c'est celle qui a trait au boisement, ou d'une façon plus large, à l'utilisation des terres incultes et dégradées, ce qu'on appelle aux Etats-Unis les " submarginal lands ».

L'activité des services chargés de la Conservation des Sols a en effet un double aspect:

$I^{\circ}$ éviter que l'utilisation agricole des sols ne conduise à leur dégradation. C'est le but des labours en ligne de niveau, des banquettes, du stubble-mulch farming, et de toutes les techniques agricoles conservatrices récemment mises au point et largement diffusées.

$2^{\circ}$ utiliser les terres abandonnées par la culture.

C'est ce second aspect qui nous intéresse plus particulièrement, puisque le service forestier français a souvent à résoudre des pro- 
blèmes de ce genre, qui rentrent à proprement parler dans ses attributions.

Nous avons vu que, dans la classification des sols en catégories par le Soil Conservation Service, on établissait une concurrence entre la vocation pastorale et forestière pour les terres non cultivables, sans donner d'ailleurs d'indication précise en faveur de l'une ou de l'autre utilisation, laissant pratiquement le choix au possesseur du sol. Pourtant; il existe des cas où l'une des solutions s'impose de préférence. Les problèmes de mise en valeur des terres incultes doivent donc être étudiés par le forestier dans leur ensemble, pour permettre de répondre dans chaque cas particulier à la question préliminaire: doit-on reboiser, préconiser des améliorations pastorales, cu des pratiques agricoles conservatrices? Il importe pour cela de savoir dans quel cadre écononique et administratif s'effectue la mise en valeur des terres incultes, et quelles sont les possibilités techniques. Nous allons examiner sous cet angle quelques exemples étudiés sur le terrain. Nous essaierons ensuite de dégager les principes qui ont guidé le choix des solutions pratiques.

Nous savons que les problèmes de Conservation du sol sont assez différents dans l'Est et dans l'Ouest des Etats-Unis, suivant l'évolution de la colonisation, et en fonction des conditions physiques, en particulier du climat.

\section{I}

Les conditions caractéristiques de l'Est se rencontrent dans la vallée du Muskingum (Ohio), région de climat tempéré, avec une lame d'eau annuelle de roo $\mathrm{cm}$. C'est une zone de dépôts glaciaires au relief mollement ondulé. Cette région, primitivement couverte de forêts feuillues, a été colonisée de façon massive vers I880. L'érosion a été provoquée d'abord par l'exploitation de nombreux gisements de houille, exploitation faite à ciel ouvert, et par le déboisement - puis par le défrichement et la mise en culture. Tout cela a amené des perturbations sérieuses dans le régime du Muskingum: les premières inondations datent de I884. Celle de I9I3 est restée célèbre pour avoir fait 500 victimes.

Pendant longtemps les habitants de la vallée ont cherché en vain un remède à ce fléau. Ce n'est qu'en 1933, grâce aux facilités exceptionnelles fournies par la création du Civilian Conservation Corps, qu'ils purent constituer un " district de conservation ", organisme distinct, en dépit du nom, de ceux qui allaient être créés peu après par le Soil Conservation Service, mais typiquement américain: Société de mise en valeur et d'intérêt public s'interdisant tout bénéfice (Public non profit Corporation), dont le but était. la lutte contre les inondations. Ce but préliminaire fut assez vite atteint 
par la construction, sous la direction du Génie de l'Armée, et grâce aux crédits et à la main-d'œuvre du Civilian Conservation Corps, de barrages de retenue constituant une dizaine de lacs réservoirs. Puis le district a fait empoissonner les lacs, a organisé des stations de canotage, des baignades, jetant ainsi la base d'un mouvement de tourisme de plus en plus important. Mais son activité devait bientôt s'étendre à la mise en valeur de l'ensemble de la région.

La vallée du Muskingum, qui est très étendue, puisque le " district de Conservation i) couvre plus de 2000000 d'hectares, soit le cinquième de l'Etat d'Ohio, est un pays de petite propriété et n'a pas une agriculture particulièrement prospère. On note sur les pentes de nombreux champs plus ou moins couverts de broussailles, abandonnés après le passage de la vague colonisatrice des fermiers en marche vers l'Ouest, et dont on évalue l'étendue à près de 800 ooo ha.

Le maintien des agriculteurs sur leurs exploitations souvent peu rentables et menacées par l'érosion a fait l'objet de tous les soins du Soil Conservation Service, appelé à donner ses conseils techniques au "District ), qui a établi à Coshocton une remarquable station expérimentale. On conseille l'extension des pâtures et de l'élevage en vue de la création d'une économie semi-pastorale. Le reboisement, moins immédiatement rentable que l'élevage, n'est préconisé dans les exploitations que là où le sol a complètement disparu, pour des pentes supérieures à $25 \%$ où le danger d'érosion dî̀ au surpâturage est trop grand. Les terres abandonnées, au contraire, qui font retour à l'Etat lorsque l'impôt foncier cesse d'être payé, sont systématiquement reboisées par le district.

Les travaux ont commencé aux environs de New-Philadelphia, et ont atteint très vite un rythme rapide, grâce à la mise au point de la charrue planteuse Garrit, qui permet de mettre en place 8 oon plants par jour, et abaisse le coût des travaux de plantation à io \$ par acre, soit I 5 par acre avec les plants. Les plants sont installés sur le bourrelet de sillons qui suivent les lignes de niveau. On a d'abord essayé de rétablir les essences constitutives de la forêt primitive, qui étaient bien connues: $2 / 3$ de chênes, I/3 de tulipier, puis on s'est aperçu que cela était impossible sans passer par le stadé préliminaire d'une essence colonisatrice. On plante donc des pins, et on constate plus tard l'installation naturelle des chênes et des tulipiers dans les peuplements ainsi constitués.

\section{II}

A Watkinsville, en Géorgie, dans le Piémont des Appalaches, le problème des terres abandonnées se pose de façon peu différente, mais sous un climat qui aggrave le risque d'érosion. Les sols d'arènes granitiques ont été épuisés par la monoculture du coton prati- 
quée de I795 à I930. C'est l'ancien domaine des plantations à maind'œuvre noire. Aujourd'hui, elles se sont morcelées, et les exploitations moyennes sont de 70 à 100 acres. Les sols sont érodés, épuisés, et $20 \%$ environ des anciennes terres agricoles ne sont plus cultivées. On cherche à donner aux cultivateurs un niveau de vie décent leur permettant de rester sur place, de ne pas abandonner leurs exploitations. On conseille de mettre en pâture les terres abandonnés, afin d'orienter l'économie agricole vers l'élevage. Les stations expérimentales étudient la mise au point de rotations de cultures. On ne reboise que là où le sol superficiel a complètement disparu.

Encore essaie-t-on de créer, grâce au $K u d z u$, liane introduite du Japon vers 1900, des pâturages médiocres dans les zones les plus ravinées. On admet qu'en bien des points la forêt aurait plus d'intérêt que le pâturage pour régulariser le régime des eaux, mais les considérations économiques et humaines priment: il faut éviter de réduire la surface fournissant des produits agricoles. Il n'en reste pas moins que la forêt a envahi naturellement d'importantes surfaces abandonnées par la culture, mais il ne se semble pas y avoir de politique de reboisement. On ne peut que le regretter.

\section{III}

En novembre 1951, dans les Appalaches, à Knoxville, capitale de la célèbre T.V.A., les eaux du fleuve Tennessee étaient chargées de boue et de limon. La T.V.A. n'a pas réussi, en effet, à supprimer l'érosion. Créée en 1933, et couvrant 26 millions d'acres, soit l'essentiel du bassin de réception du fleuve, elle a réglé le problème des inondations, naguère très fréquentes, par la construction de 17 digues. Bien que les retenues ainsi créées aient inondé irrémédiablement les meilleures terres de la vallée, il a paru plus expéditif et plus économique de corriger le régime des eaux par des ouvrages placés dans le lit du fleuve que d'essayer de remédier au ruissellement sur les pentes. Ceci est caractéristique de l'état d'esprit des constructeurs de barrages qui ne songent qu'exceptionnellement à travailler au maintien du sol dans les parties supérieures des bassins de réception.

La situation des cultivateurs a néanmoins été rendue plus prospère par la fourniture à bas prix d'énergie électrique et d'engrais grâce aux usines installées pour-utiliser l'énergie des barrages. Nombre d'entre eux trouvent dans les entreprises industrielles des occupations de complément. Bien que la conservation des sols leur soit enseignée, ils n’ont pas délaissé les pratiques culturales génératrices d'érosion, trouvant grâce à l'industrie locale le complément de ressources qui leur a permis de ne pas abandonner leurs terres. Au demeurant, le bassin du Tennessee est relativement peu peuplé et 
les forêts occupent encore $50 \%$ de sa superficie. Il n'est' guère question de reboisement jusqu'à présent.

On ne peut qu'émettre des réserves sur les solutions type T.V.A.: la mise en valeur d'un bassin fluvial comporte normalement la conservation des sols et de ses versants.

IV

En Oklahoma, dans l'ancienne Prairie centrale, le bassin •de Sandstone Creek contribue de façon importante aux crues torrentielles du fleuve Wachita. Il est constitué par des terrains d'âge permien, mollement ondulés, mais creusés de multiples ravins à bords verticaux: l'homme a créé, en 50 ans, un relief jeune dans ces terrains anciens, en cultivant l'arachide et le coton. L'érosion est toujours active, alimentée par les pluies torrentielles, car la lame d'eau annuelle de $600 \mathrm{~mm}$ est très irrégulièrement répartie. La solution raisonnable serait l'interdiction de toute culture sur les terrains érodés, la mise en pâturage contrôlé, sans surcharge, avec plantations de saules et de peupliers dans les ravins. Malheureusement, il n'est pas question en période de prospérité économique, de limiter trop l'utilisation d'un sol qui n'est pas complètement épuisé.

Or, ce sol est très friable. La technique des banquettes même n'est pas loin d'être abandonnée: elles sont trop souvent emportées par les pluies ou donnent naissance à de nouveaux ravins. On a essayé naguère de boucher les ravins par des barrages élémentaires en maçonnerie inspirés de ceux qui ont été mis au point dans les Alpes françaises. Ils ont été emportés, faute d'ancrage solide. Aujourd'hui, on traite le ravinement par des digues en terre battue créant des retenues d'eau au moment des orages, ou par des levées de terre destinées à détourner les eaux des ravins. Les digues de retenue font songer aux levées d'étang en argile corroyée, créées au Moyen Age dans notre pays.

Comme dans tout le Middle West, le reboisement est limité à la constitution de brise-vents. On utilise maintenant des résineux: $P i$ nus laricio anstriaca, Pinus ponderosa, Juniperus virginiana, à la place des feuillus primitivement en faveur: Robinia pseudoacacia, Gleditschia triacanthos, Eleagnus angustifolia, Chilopsis linearis, Celtis occidentalis, Maclura pomifera, Morus alba var. tatarica.

Contraste saisissant de paysage dî̀ à une action différente de l'homme: à quelques dizaines de kilomètres de Sandstone Creek se trouve le refuge de bisons de Wichita, établi sur des terrains granitiques de basses montagnes, aussi érodibles que ceux de Sandstone Creek et sous un climat analogue. Un début d'érosion y a été observé en I935-37, dû à la surabondance des animaux; et la surcharge des pâtures s'est traduite alors par l'apparition d'une végétation buissonnante et surtout de chênes. Mais on a limité le pâtu- 
rage et il n'y a plus maintenant trace d'érosion: preuve évidente qu'une utilisation prudente du sol, par l'élevage, serait la meilleure solution pour la remise en état des terrains érodés de Sandstone Creek.

\section{V}

La région de Dalhart (Texas) est comprise dans cette partie de la Prairie qui, sous un climat excessif, a été érodée par, la monoculture du blé, au point d'aboutir à la formation de dunes continentales. C'était le dustbow. Vers I937, le gouvernement a racheté d'importantes étendues de terres abandonnées, dans le cadre de la politique de " resettlement ), et, depuis, le Soil Conservation Service a réussi un travail remarquable de fixation des dunes et de reconstitution des pâturages.

Une technique de fixation des dunes continentales a été mise au point. Les dunes sont dues à l'action du vent qui emporte l'argile et les limons des sols (apparentés au type châtain des steppes) désagrégés.et desséchés. Le sable reste. Poussé par le vent, il s'accumule le long d'obstacles quelconques: buissons, clôtures, où se rassemblent, déracinés par le vent et desséchés, les débris de Salsola pestifer, Salsolacée introduite devenue envahissante dans les terrains dégradés. Les dunes progressent ensuite de façon classique en détruisant devant elle la végétation par le vent chargé de grains de sable.

Pour fixer la dune, on commence par arrêter l'érosion dans la zone d'alimentation en y interdisant culture et pâturage. On cherche ensuite à écréter la dune à la niveleuse, ou en créant des sifflevents à l'aide de panneaux ou de sacs de sable. En même temps, on trace des sillons parallèles autour d'elle pour capter le sable qui aurait tendance à progresser. C'est ce qu'on appelle le " listing ". La dune, une fois réduite de hauteur, est ensemencée avec des graminées (Andropogon hallii, Boutelouea curtipendula. Sorghastum nutans, entre autres).

Les sols seulement érodés, où il reste encore du limon, peuvent ètre traités aisément par le réensemencement seul. Certaines dunes même se sont fixées spontanément avec une végétation de yuccas et de graminées.

Les terrains corrigés et fixés, après rachat par le gouvernement, ont été mis en pâture, avec un système de concession par permis, à raison d'une tête de bétail par 8 acres (3,20 ha), Ce système a assuré le maintien'en bon état du sol reconstitué. Il a donné lieu toutefois à de nombreuses protestations de cultivateurs qui désiraient remettre en culture ces terrains fragiles, tant la nature humaine est imprévoyante. D'ailleurs, le bon exemple des terres domaniales n'est pas partout suivi. Certains fermiers s'obstinent à cultiver des sols dont la vocation est au plus pastorale, et l'érosion éolienne sévit alors, met- 
tant en danger les terres voisines. Sur les propriétés privées mises en pâtures, le danger de surcharge en bétail subsiste: aucune loi n'impose, dans l'intérêt général, des limitations à l'utilisation du sol. Si l'essentiel de l'érosion a été arrêté, si des résultats magnifiques ont été obtenus, ils ne sont donc ni complets, ni définitifs.

Dans tout le Texas, les plantations brise-vent sont rares, en raison de la sécheresse ( 25 à $50 \mathrm{~cm}$ de pluies par an). Cependant, on en a constitué quelques-unes avec des Tamarix. C'est le pays du " $d r y$ farming ), que l'on tend à remplacer par le "stubble-mulchfarming ", et des banquettes, qui sont classiques sur les pentes de 2 à $3 \%$. Mises à part les régions très érodées, au sol particulièrement fragile, comme celle de Dalhart, la culture du blé est la règle avec celle du Sorgho. Les pâturages ne subsistent que sur les terres les plus médiocres, pour l'élevage du bétail Hereford.

\section{VI}

Dans la région montagneuse de l'Ouest, que l'on appelle parfois le Grand Désert Américain, la plus grande partie du territoire est couverte d'une véritable brousse, le (( range ), qui est utilisé comme terrain de parcours pour le bétail. Le sol est couvert de maigres graminées avec de nombreuses touffes de végétaux buissonnants, dont l'un des plus communs est le "Sagebrush », Purshia tridentata.

Bien souvent cette forme de végétation est le résultat de la dégradation, sous l'effet du pâturage, de pelouses xérophiles dont les graminées vivaces ont disparu pour faire place à des graminées an- nuelles. On y note certaines espèces méditerranéennes importées par les Espagnols et devenues envahissantes, comme Bromus tectorum. Parfois, elle couvre des sols naguère couverts de forêts, sur les pentes Ouest de la Sierra Nevada, où le reboisement en Pinus ponderosa est encore possible, et à coup sûr souhaitable si l'on considère le régime torrentiel des cours' d'eau.

La dégradation du sol par l'érosion, qui peu à peu déchausse les plantes, est évidente dans les immenses étendues du ( range ): elle a ému les pouvoirs publics. Le reboisement est envisagé, mais on n'a entrepris que peu de choses dans ce domaine. L'effort a porté jusqu'ici, et depuis 1933, sur le rétablissement de la prairie dans les territoires broussailleux et une technique spéciale, celle du réensemencement a été mise au point. Elle consiste à briser la compacité du sol et à détruire les buissons par le passage d'une lourde charrue qui travaille sur une profondeur de 7 à $\mathrm{ro} \mathrm{cm}$. Il est essentiel en effet d'éliminer la concurrence pour l'eau des buissons, au système radiculaire développé. Puis on sème au semoir en lignes, au début de la saison humide. On utilise des Agropyron et Elymus sous les climats à pluviosité hivernale, des Eragrostis, Andropogon, Boulelouea, sous 
les climats à pluies d'été. On met en défens pendant deux ans au moins, pour permettre l'établissement de la prairie.. Le coût de l'opération est peu élevé: Io \$ à l'acre environ. Cette technique semblerait pouvoir être adaptée pour la mise en valeur de certains sols méditerranéens.

\section{VII}

Le territoire surpeuplé de la réserve des Indiens Navajos, en Arizona, pose en réduction les problèmes Nord-Africains. Il y avait en I870 sur la réserve, qui couvre 25000 milles carrés, une dizaine de milliers d'Indiens pasteurs. Grâce aux progrès médicaux, ils sont maintenant environ 65000 . Le troupeau s'est accru en proportion des besoins de la population, et d'autant plus vite qu'il est considéré comme un signe de richesse sociale.

Il en est résulté une érosion intense. On a dî réduire le troupeau par voie d'autorité, ce qui a incité un certain nombre d'Indiens à quitter la réserve pour aller dans les villes chercher leur subsistance, comme manœuvres ou garçons d'ascenseurs. On a essayé d'appliquer la technique de réensemencement à la rénovation du pâturage, mais elle n'a pu réussir à cause de la sécheresse et du maintien d'une charge excessive en bétail.

La solution reste à trouver. Quelle qu'elle soit, elle suppose la mise en défens d'une partie importante du pays Navajo, ce qui ne manquera pas de soulever des difficultés politiques.

\section{VIIJ}

La lutte contre l'érosion dans la vallée du San Fernando, près de Los Angeles, fait songer aux problèmes du Midi Méditerranéen. Le surpâturage sur les pentes du bassin de réception du fleuve a provoqué des inondations de plus en plus fréquentes. Comme c'est l'habitude, on a construit à grands frais de nombreuses digues de retenue, canalisé le lit de plusieurs torrents, sans s'attaquer à l'origine des perturbations constatées dans le régime des eaux, à savoir la dégradation des pentes. Faute d'une législation appropriée, il est d'ailleurs à peu près impossible d'envisager des travaux de fixation sur de nombreux versants qui sont propriétés privées et sont soumis aux abus de pâturage. Jusqu'en I95I, le Soil Conservation Service n'a pu travailler que sur des terrains achetés par une Compagnie pétrolière en vue de la réalisation de forages, et soustraits par elle à tout pâturage, mais les travaux réalisés ont un certain intérêt technique.

Les précipitations atmosphériques annuelles sont de l'ordre de grandeur de $400 \mathrm{~mm}$ et se produisent surtout en hiver. On a d'abord tenté des reboisements en robinier, Pinus canariensis, Cupressus arizonica. On n'obtient pas assez rapidement le couvert nécessaire pour 
fixer le sol en pente. On a recours maintenant au gazonnement et $\dot{a}$ la plantation de broussailles. On diminue d'abord le ruissellement superficiel par l'épandage d'une couverture morte de débris divers, paille, foin, feuilles mortes, et on plante des boutures de Baccharis viminea, dont la facilité de reprise rappelle celle de nos saules. On installe des végétaux buissonnants, tels que Ceanothus thyrsiflorus, Pyracantha yunnanensis, Rhus ovata, préparés en mottes en pépinière dans les cylindres de papier goudronné. On sème des graminées: Panicum antidotale, Stipa cernua, Bromus carinatus, entre autres.

Pour fixer le sol préalablement aux travaux, on a mis au point une technique qui consiste à répandre, en même temps que les graines de graminées, une pulvérisation de bitume. Ce revêtement protecteur se désagrège au moment de la germination. Mais le plus souvent et sur les pentes particulièrement érodibles, on opère de la manière suivante: on trace des sillons de $I 5$ à $25 \mathrm{~cm}$ de profondeur suivant les lignes de niveau, tous les 2 mètres environ, et on $\mathrm{y}$ instálle des bourrées ou des branchages, fixés de place en place par des piquets, et à demi recouverts de terre. On constitue ainsi de petites banquettes. On plante et sème dans l'intervalle entre deux sillons.

\section{IV. - Conclusions}

D'après les exemples qui précèdent, on voit que l'utilisation, la remise en valeur des terres " submarginales » érodées et abandonnées tient compte de plusieurs facteurs, leś uns d'ordre physique, les autres d'ordre technique, et d'autres enfin d'ordre économique. C'est en fonction de ces éléments qu'on décide de procéder au boisement ou à la remise en pâture.

En ce qui concerne les facteurs physiques, on sait que la végétation forestière est limitée par l'altitude et la sécheresse, que certaines formations boisées ouvertes (woods), et le maquis, sont adaptés à des conditions xérothermiques qui excluent la forêt. Ainsi, dans des conditions de sécheresse excessive, il sera vain de chercher à boiser: on n'obtiendra pas mieux qu'une brousse d'arbustes buissonnants sur certains versants rongés par le soleil. Du point de vue des ressources en eau, si précieuses pour l'irrigation, le couvert ainsi constitué évaporera modérément, ce qui est un avantage. Mais ailleurs il apparait que la formation d'un sol perméable, et susceptible de supprimer le ruissellement, est très lente sous un couvert d'herbes; que sa dégradation est fréquente sous l'influence de nouveaux abus de culture ou de pâturage. Alors on cherche à consolider par le boisement les résultats du réensemencement si l'on craint le surpâturage. Il est certain que la forêt, une fois établie, est plus facile à défendre, moins facile à dégrader que la pâture: on est mieux assuré de sa permanence. Toutefois, les formations buissonnantes et 
herbacées semblent souvent résister mieux à l'incendie que les peuplements artificiels d'essences colonisatrices. Les arbustes de Californie rejettent après le passage du feu, les arbres meurent. Seuls certains pins de Floride sont adaptés à l'incendie $\left(^{*}\right)$. mais à un degré moindre que Sabal palmetto qui les accompagne.

Les considérations techniques introduisent d'autres limitations qui sont souvent question de prix de revient: le réensemencement est sensiblement moins onéreux que le reboisement. Mais le reboisement. est possible à moindres frais sur des pentes escarpées, où l'utilisation de l'outillage mécanique nécessaire au réensemencement est impossible. De façon générale, la pente a une importance primordiale pour la détermination de la destination à donner au sol et pour le choix d'une technique d'amélioration.

Les conditions économiques sont bien entendu fort importantes aussi. L'administration chargée de la mise en valeur des terrains incultes se pose la question de savoir ce dont a besoin l'économie locale: du bois, ou bien de la viande et du lait, telle est en gros l'alternative. L'intérêt de certains boisements pour le tourisme et pour la chasse, entre fréquemment en ligne de compte. Le mode d'utilisation des sols remis en état est examiné : pourra-t-on créer des exploitations rentables avec des sols fragiles, des pâtures qui ne pourront être qu'extensives ? Pratiquement, on fait l'impossible pour maintenir sur place les cultivateurs, au prix bien souvent d'un contrôle insuffisant de l'érosion. On reboise les terres abandonnées par la culture et l'élevage après complète dégradation dans certaines régions, mais le reboisement est loin d'être généralisé.

Dans le domaine des recherches, la notion de forêt de protection, indispensable à la défense du sol contre les érosions ou à la régularisation du régime des eaux est pourtant en passe d'être redécouverte outre-atlantique, voire reprise avec plus d'ampleur qu'en Europe, en considération de l'influence de la forêt sur le climat et les inondations. Seul le reboisement permet d'être assuré que certains secteurs montagneux ne seront plus soumis à des formes d'agriculture ou d'élevage qui, pour de maigres profits, risquent de détruire par éboulements, inondations, dépôt de matériaux grossiers, des exploitations agricoles ou des industries prospères situées dans les vallées.

L'influence de la forêt sur le régime des eaux a été particulièrement étudiée aux Etats-Unis, où la sauvegarde des ressources en eau vient au premier plan de la politique de Conservation. Le rôle joué par la couverture végétale du sol vis-à-vis du ruissellement a été étudié méthodiquement sous différentes cultures et sous la prairie,

(*) Voir à ce sujet l'étude de P. Bouvarel: « Les Pins du Sud-Est des Etats-Unis 》. Rev. For. Franc., septembre 1953, p. 585. (N.D.L.R.). 
par la méthode lysimétrique notamment, dans la Station du Soil Conservation Service, à Coshocton (Ohio). Pour la forêt, il est difficile de concevoir des lysimètres satisfaisants. On a étudié ce qui se passait sur l'ensemble d'un versant montagneux, en mesurant à l'aide de jauges le débit des ruisseaux qui y prennent naissance et à l'aide de pluviomètres les précipitations qui s'y produisent. Parfois on a ajouté des puits pour se rendre compte des mouvements de l'eau dans le sol. Les premières études ont été réalisées à Wagon Wheel Gap, dans le Colorado. Elles ont été suivies de nombreuses autres, en particulier à San Dimas (Californie) où l'on a mis en évidence l'action de l'incendie: par destruction de l'humus, la perméabilité du sol est diminuée. Le ruissellement et l'érosion augmentent considérablement sous l'effet de la pratique de l'incinération annuelle.

Des études réalisées récemment à Asheville, dans les Appalaches, ont permis de préciser l'action du surpâturage: il y a tassement du sol par le piétinement, d'où diminution de la vitesse d'infiltration de l'eau qui peut être réduite de $90 \%$ dans certains cas, d'où encore accroissement des eaux de ruissellement, génératrices d'érosion. Lorsque le pâturage s'exerce en forêt, le tassement du sol diminue la croissance des arbres, cette diminution pouvant aller jusqu'à $50 \%$. De plus, le pâturage dégrade la forêt par élimination sélective des semis de certaines essences.

A Asheville encore, on a précisé l'action régulatrice de la forêt sur l'écoulement des eaux: la protection du sol par la couverture morte lui permet d'absorber l'essentiel des précipitations atmosphériques. Sur les pentes, il y a ensuite écoulement ralenti vers le bas dans la partie superficielle du sol: la forêt abaisse donc les maxima de débit et prolonge la période de crue des ruisseaux qu'elle engendre. Cette action régulatrice se fait aussi sentir en temps de neige: la forêt en ralentit la fusion, et une partie des eaux qui en proviennent est absorbée par le sol. Les expériences réalisées ont permis de préciser ces idées générales, déjà connues en Europe dans les grandes lignes, par des masses de faits, de documents et d'observations.

Cependant, l'action du revêtement forestier des pentes des bassins de réception a été contestée par les Ingénieurs constructeurs de barrages: ils ont mis l'accent sur l'évaporation de l'eau par la forêt, qui équivaut à une perte en pays sec. Bien qu'une partie de cette eau soit souvent précipitée à nouveau sur place, cette action est indéniable, et l'on recherche actuellement s'il est possible de procurer au sol un couvert végétal buissonnant qui, sans évaporer autant que la forêt, lui permettrait de conserver ses qualités et d'éviter l'érosion. Mais, en pays tempéré, la forêt apparaît comme le meilleur couvert possible du point de vue de la régularisation du régime des eaux, sans parler de l'intérêt de la fourniture d'une eau claire et 
limpide, mis en évidence à maintes reprises. C'est le rôle, reconnu depuis un siècle en Europe, des forêts de protection dans les secteurs de montagne.

L'influence de la forêt sur le climat local est encore aux EtatsUnis un sujet de controverse malgré de nombreuses expériences réalisées à ce sujet. D'autres pays nous vient la notion que la dévastation de la forêt sur de vastes étendues entraine un asséchement du climat local, une descente de la nappe phréatique. Mais cette constatation, qui expliquerait le caractère subclimacique du chapparal californien, adapté à la sécheresse et à l'incendie et qui a succédé à la forêt en de nombreux points depuis un siècle, n'est généralement pas admise.

Des rapides indications qui précèdent, il résulte que les EtatsUnis, obligés de faire face à des dégradations exceptionnellement rapides dues à la colonisation, ont accumulé une expérience considérable dans la pratique de la restauration et de la conservation des sols.

Qu'a-t-elle d'utilisable pour nous autres Européens ?

Certes, rien n'est directement applicable en raison des différences de sol et de climat. Ne répétons pas l'erreur des premiers colons qui ont voulu imiter les modes de culture de leurs pays d'origine; ne cherchons pas, à notre tour, à calquer les méthodes de guérison des sols en usage outre-atlantique. Mais nous pouvons retenir certaines idées.

Dans le domaine technique, des méthodes de fixation des pentes, de lutte contre le ravinement qui ont été rapidement décrites dans ce qui précède, sont peut-être susceptibles d'adaptation : mais elles sont utilisées très généralement sur des pentes moins fortes que celles sur lesquelles le service forestier français travaille en montagne. L’idée de réensemencer en graminées, après labour, les formations végétales buissonnantes analogues à la brousse ou à un maquis clair paraît susceptible d'application dans le Midi méditerranéen.

C'est surtout en matière, non plus de technique, mais de politique gouvernementale que l'expérience américaine apporte des enseignements. L'un de ceux-ci est la difficulté d'une politique de "resettlement ) en pays démocratique, même lorsqu'on déplace les gens pour élever leur niveau de vie, faire en quelque sorte leur bonheur malgré eux. Par contre, le rachat des terres déjà abandonnées, par l'Etat, ou mieux par des Sociétés de reboisement ou de mise en valeur, comme dans la vallée du Muskingum, semble être une excellente mesure. Le "Zoning », appliqué en dehors des centres urbains aux forêts, pâtures ou champs cultivés, peut être un moyen progressif d'obtenir dans certains cas le remaniement de la structure des collectivités rurales. Mais le contrôle de terrains érodés dans l'intérêt public, 
voire leur expropriation, rendus possibles par les lois françaises sur ia Restauration des terrains en montagne, n'existent pas aux EtatsUnis. Pourtant, l'opinion y est répandue que l'utilisation de certains terrains présente des dangers pour la collectivité, que l'appropriation privée de certains sols n'est pas souhaitable.

En ce qui concerne la pratique administrative, la grande leçon du succès du Soil Conservation Service est qu'en matière de lutte contre l'érosion, comme en beaucoup d'autres, on obtient surtout des résultats par la persuation et l'enseignement. Rien n'a été négligé dans ce domaine: il est difficile de se faire une iclée du nombre de brochures, d'articles de journaux, de conférences, consacrés à la Conservation des Sols.

Aussi le mouvement de Conservation du Sol aux Etats-Unis a eu un résultat important, celui d'avoir éveillé l'opinion, non seulement américaine, mais mondiale, à la compréhension d'un problème essentiel. On sait maintenant quelle est l'action de l'homme dégradant les sols sous des climats tropicaux ou en pays neuf. Il est hors de doute que les colons européens en Afrique sont, eux aussi, responsables de bien des catastrophes. Plus près de nous, l'histoire des sols du Midi Méditerranéen et de l'Afrique du Nord révèle bien la même emprise destructrice. Le processus est plus lent qu'en Amérique, mais il existe. Et la dégradation des sols est, on le sait par l'histoire. susceptible de se révéler fatale à certaines civilisations. L'idée de conservation du sol, dans le cadre de la conservation des ressources naturelles, nous révèle donc l'importance de cette évolution pour l'avenir des peuples.

Enfin, l'étude des mesures prises montre l'importance du contrôle de l'utilisation des sols par l'homme pour leur restauration ou leur conservation. C'est autant par des mesures administratives prohibant la culture ou réglementant le pâturage que par des travaux de restauration qu'on parvient à un résultat et qu'on assure sa permanence. En d'autres termes, les tentatives de boisement, d'améliorations pastorales des terres dégradées, doivent, sous peine d'échec, s'intégrer dans le cadre d'une politique économique régionale ou locale, correspondre à l'intérêt des propriétaires ou des exploitants. Permettre le maintien d'exploitations équilibrées, malgré les restrictions indispensables à toute entreprise de restauration, est un des principaux soucis des administrations d'outre atlantique.

En présence d'un terrain érodé et inculte susceptible d'être boisé, la première question de bon sens qui se pose est donc: comment est-il utilisé ? - la seconde: à quoi pourra-t-il l'être une fois restauré ? Chez nous, le pâturage ou la chasse grèvent bien des terres abandonnées d'une servitude qui rend malaisée leur mise en état de production. Il est des utilisations incompatibles dans une certaine 
mesure : chasse et pâturage, pâturage et forêt, garenne et forêt, tourisme et culture.

Ce point de vue fait entrevoir l'intérêt qu'il y aurait à étudier scientifiquement les rapports entre l'homme et le sol sous le signe de l'histoire, de la géographie locale, des sciences agricoles et forestières, pour déterminer l'évolution de son utilisation dans le passé ainsi que les mesures qu'il est souhaitable et possible de prendre dans l'avenir pour assurer l'exploitation conservatrice la plus productive.

On rejoint ici les conclusions de ceux qui s'intéressent à la Protection de la Nature: il s'agit de définir la place que l'Homme peut y occuper, les rapports qu'il peut avoir avec elle sans la détruire, ou sans compromettre sa propre destinée en se rendant dépendant d'un milieu artificiel et instable.

H. MOREL.

Avril I953.

\section{BIBLIOGRAPHIE (par ordre chronologique)}

La bibliographie de la Conservation des Sols est aujourd'hui considérable. Seuls les ouvrages consultés pour la rédaction de cet article ont été mentionnés.

I934 - Making the best use of Wisconsin Land - publié par University of Wisconsin, Madison.

I938 - Soils and Men - Yearbook of Agriculture, Government Printing Office, Washington D.C.

I938- La terre incendiée - Essai d'Agronomie comparée, par KunnholtzLORDAT, Nîmes.

I939 - Vanishing Lands, par JACKs and Whyte, New-York.

1939 - Soil Conservation, par BENNETT, New-York.

i939 - Soil Defense of Fange and Farm Lands in the Southwest - Department of Agriculture, Washington D.C.

1940 - The story of Soil Conservation in the South Carolina Piedmont Department of Agriculture, Washington D.C.

194I - Early American. Soil Conservationists - Department of Agriculture, Washington D.C.

1942 - Regrassing for Soil Protection in the Southwest - Department of Agriculture, Washington D.C.

1943 - The American Land, par Van Dersal, New-York.

1943 - Terracing for Soil and Water Conservation, par Hamilton - Farmer's Bulletin, Washington D.C.

1944 - Natural Principles of Land Use, par Graham, New-York.

1944 - Kudzu for Erosion Control in the Southeast - Farmer's Bulletin, Washington D.C.

1944 - Afrique, Terre qui meurt, par Harroy, Bruxelles.

I945 - Man and the Soil, par Micky, Chicago.

1946 - Terrace outlets and Farm drainage ways, par Hamilton, Farmer's Bulletin, Washington D.C.

1946 - Strip cropping for Conservation and Production - Farmer's Bulletin, Washington D.C.

1946 - Reconstruction by way of the Soil, par WRENCH, Londres.

1946 - The life and death of the Land, par Whitaker, Nashville (Tennessee).

I947 - Elements of Soil Conservation, par BENNETt, New-York. 
1947 - When Drought returns to the Great plains, par DALE - Farmer's Bulletin, Washington D.C.

1947 - L'érosion du sol, par Raymond Furon, Paris.

1948 - Stubble mulch farming - Farmer's Bulletin, Washington D.C.

I948 - What is a Conservation Farm Plan ? - Department of Agriculture,

Washington D.C.

1948 - Road to Survival, par Vogr, New-York.

I948 - Our Plundered Planet, par Fairfield OsbonN, Boston.

1948 - Grass - Yearbook of Agriculture, Washington D.C.

1948 - Rural Zoning in Wisconsin - publié par University of Wisconsin, Madison.

I948 - Conservation du Sol - Etude internationale F.A.O.

r 949 - L'homme et le sol, par Prat, Paris.

1949 - Grasses and Legumes for Soil Conservation in the Pacific Northwest - Department of Agriculture, Washington D.C.

I949 - Trees and Shrubs for Erosion Control in Southern California Mountains - Department of Agriculture, Washington D.C.

I950 - Manuel de Conservation des Sols - Department of Agriculture, Washington D.C.

I950 - La politique de Conservation des eaux du Gouvernement fédéral aux Etats-Unis, par J. GotrmanN - Bulletin de l'Association des Géographes français, $\mathrm{N}^{\circ}$ s 2 TO-2I $\mathrm{I}$.

1950 - Dams are not enough par Frank et Netboy, American Forest, Washington D.C.

1950 - Conquest of the Land through seven thousand years - par Lowdermilk - Department of Agriculture, Washington D.C.

I95I - Agricultural Conservation program - National Bulletin for 195I, Department of Agriculture, Washington D.C.

I95I - T.V.A. I95I - Washington D.C.

I95I - L'érosion, par Pouguet, Paris.

195I - La Conservation du sol - Le problème français - Bulletin technique d'information des Ingénieurs des Scrvices Agricoles, Paris.

Une bibliographie plus détaillée que la précédente, mais encore incomplète, a été publiée par le Department of Agriculture, Washington D.C. en I95I (Agriculture Information Bulletin, $\mathrm{N}^{\circ} 4063$ ).

A signaler, en vutre, deux revues publiées aux Etats-Unis: Soil Conservation, organe du Soil Conservation Service, et Our Public Lands, organe du Bureau of Land Management. 